\title{
Gracilis Myocutaneous Flap: Adding to the Armamentarium of Complex Sacrococcygeal Defect Reconstruction
}

\author{
K. Preetha Rani $\quad$ J. Satish Kumar ${ }^{1} \quad$ V. Singaravelu ${ }^{1}$ \\ 1Department of Plastic Surgery, Sri Ramachandra Institute of Higher \\ Education \& Research, Porur, Chennai 600116, Tamil Nadu, India
}

\section{Fernandes Deyonna ${ }^{1}$}

\begin{abstract}
Address for correspondence K. Preetha Rani, Department of Plastic Surgery, Sri Ramachandra Institute of Higher Education \& Research, Porur, Chennai 600116, Tamil Nadu, India (e-mail: dafodilp@gmail.com).
\end{abstract}

\author{
Abstract \\ Keywords \\ - complex sacrococcy- \\ geal defect \\ - rectal cancer \\ - gracilis myocutaneous \\ flap
}

Background With increasing radicality of rectal cancer surgeries, the postoperative defects are becoming more complex. This demands an ideal reconstructive option with minimal morbidity to the patient. Although vertical rectus abdominis myocutaneous (VRAM) flap is the commonly used flap, gracilis myocutaneous flap is increasingly being performed to avoid morbidity associated with VRAM flap.

Results We share our experience about two of our patients treated for rectal malignancy with pelvic exenteration who were reconstructed immediately with pedicled gracilis myocutaneous flap. Both the patients had an uneventful postoperative recovery period and were discharged on postoperative day 10 . During follow-up period both patients had a healthy flap with no evidence of recurrence. No perineal hernias or gross dehiscence of skin closure occurred.

Conclusion Gracilis myocutaneous flap has its own place with unique advantages adding to the armamentarium of reconstructive options for complex perineal defects, thereby avoiding the morbidity associated with VRAM flap. It stands as a reliable alternative in patients where VRAM cannot be used.
Two patients underwent pelvic exenteration including resection of sacrum for rectal malignancies. One a locally recurrent rectal cancer and the other a primary case, rendering a large defect which was reconstructed with bilateral and unilateral pedicled, gracilis myocutaneous flap, respectively. Postoperatively the patient was nursed in the prone/lateral position for 2 weeks.

\section{Case 1}

Our first patient was a 55-year-old man who presented with a large locally recurrent disease involving the sacrum following an abdominoperineal resection and radiation therapy for a primary rectal cancer, after a disease-free interval of 8 years. The patient underwent an extended pelvic exenteration involving removal of sacrum with an end colostomy and an ileal urinary conduit, rendering a complex three-dimensional (3D) defect bounded anteriorly by pubic bone and pelvic side wall laterally measuring $18 \mathrm{~cm}$ in length, $10 \mathrm{~cm}$ in width, and $15 \mathrm{~cm}$ in depth. The defect was reconstructed using a bilateral gracilis myocutaneous flap; the first flap was de-epithelized and used to fill the pelvic cavity and the second flap was stacked over it as skin cover. The superficial flap suffered partial pressure injury due to nonadherence to nursing care in prone position and necessitated subsequent debridement and skin closure (-Fig. 1).

\section{Case 2}

Our second patient was a 58-year-old man who had a locally advanced rectal cancer invading the sacral fascia and perianal tissue. Patient was treated with neoadjuvant chemoradiation followed by extra-levator ani abdominoperineal excision along with overlying sacrum. The 3D surgical defect was bounded anteriorly by bladder and laterally by pelvic side
DOI https://doi.org/

$10.1055 / \mathrm{s}-0039-1696078$ ISSN 0970-0358.
License terms

(우 (1) $\Theta \circledast$ 

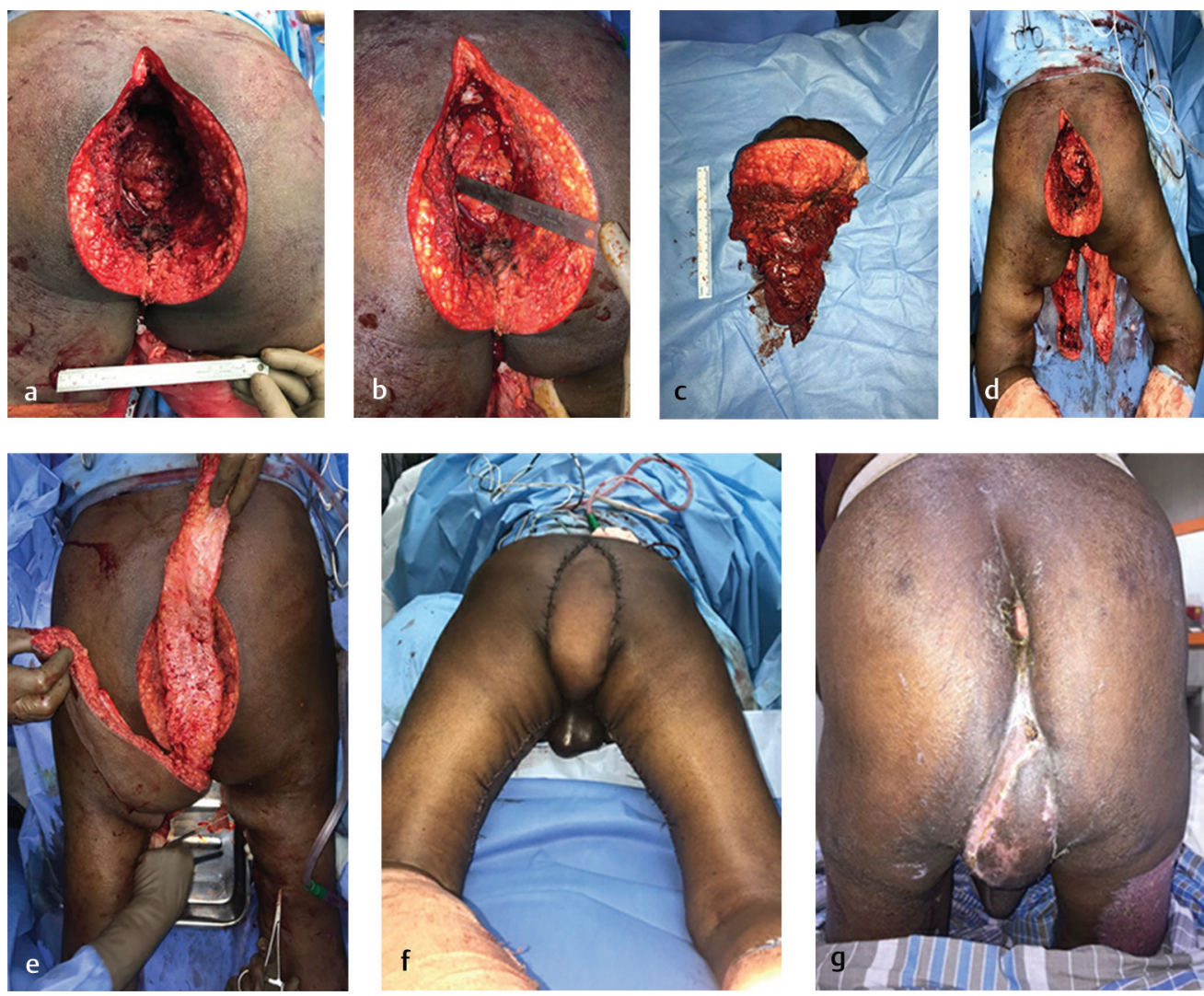

Fig. 1 (a-c) Defect post abdominosacral resection. (d) Bilateral gracilis myocutaneous flap harvested in supine on the major pedicle. (e) In prone position, abdominal cavity protected with mesh and muscle flaps tunneled and placed one over the other after de-epithelization. (f) Immediate postoperative status. (g) 3 months postoperative status.

walls measuring $16 \mathrm{~cm}$ in length, $8 \mathrm{~cm}$ in width, and $10 \mathrm{~cm}$ in depth. A unilateral gracilis myocutaneous flap was used for reconstruction (-Fig. 2).

\section{Surgical Technique}

Reconstruction was done following pelvic exenteration, ostomy creation, and abdominal closure. Omental flap was mobilized and placed in pelvis followed by mesh placement to prevent herniation of abdominal contents. In the first case, bilateral gracilis myocutaneous flap was planned with a broad skin paddle that was centered over the muscle. It extended upto distal third of thigh with a maximum width of $12 \mathrm{~cm}$ proximally and tapering distally for a length of $18 \mathrm{~cm}$. In the second patient, skin paddle was taken with a maximum width of $10 \mathrm{~cm}$ proximally and tapering distally for a length of $16 \mathrm{~cm}$. With the patient in supine position and legs abducted, incision was made posterior to adductor longus to expose gracilis muscle belly, without separating the cutaneous portion from the proximal portion of muscle. Dissection was done from the distal to proximal direction in the avascular plane between adductor longus and gracilis muscles sacrificing distal minor pedicles and retaining the dominant proximal pedicle. Proximal elevation of skin paddle was done in continuity with gracilis and temporarily sutured to muscle to prevent disruption of perforating blood vessels. The flap was mobilized by proximal pedicle dissection upto the entry of major pedicle into the gracilis muscle, pivoted at that point and tunneled in subcutaneous plane. After repositioning the patient into prone position, flap was inset into the defect. Reach of the flap posteriorly till L4-L5 level achieved. Postoperative care continued in prone position to prevent pressure injury to the flap.

\section{Discussion}

Rectal cancer is increasingly being managed with multimodal treatment to improve survival. But surgical resection remains the cornerstone of treatment. In patients with locally advanced disease, radical surgical resections are being performed increasingly leaving complex challenging surgical defects to the reconstructive surgeons. Recurrent rectal cancers are more aggressively resected with the curative intent, further adding to the complexity of the issue. Use of radiation therapy in the preoperative setting for carcinoma of rectum increases the chance of impaired wound healing following reconstruction. Size of the defect combined with rigid bony pelvis favors fluid accumulation which may interfere with wound healing. ${ }^{1}$ Ours is one such experience where large 3D surgical defects was given to us for reconstruction.

Options available to us were the gracilis flap (gracilis muscle flap, gracilis myocutaneous flap), rectus abdominis flap (vertical rectus abdominis myocutaneous [VRAM] flap, oblique or transverse rectus abdominis myocutaneous flap). The rectus abdominis flap often has been favorably described in literature.,2,3 

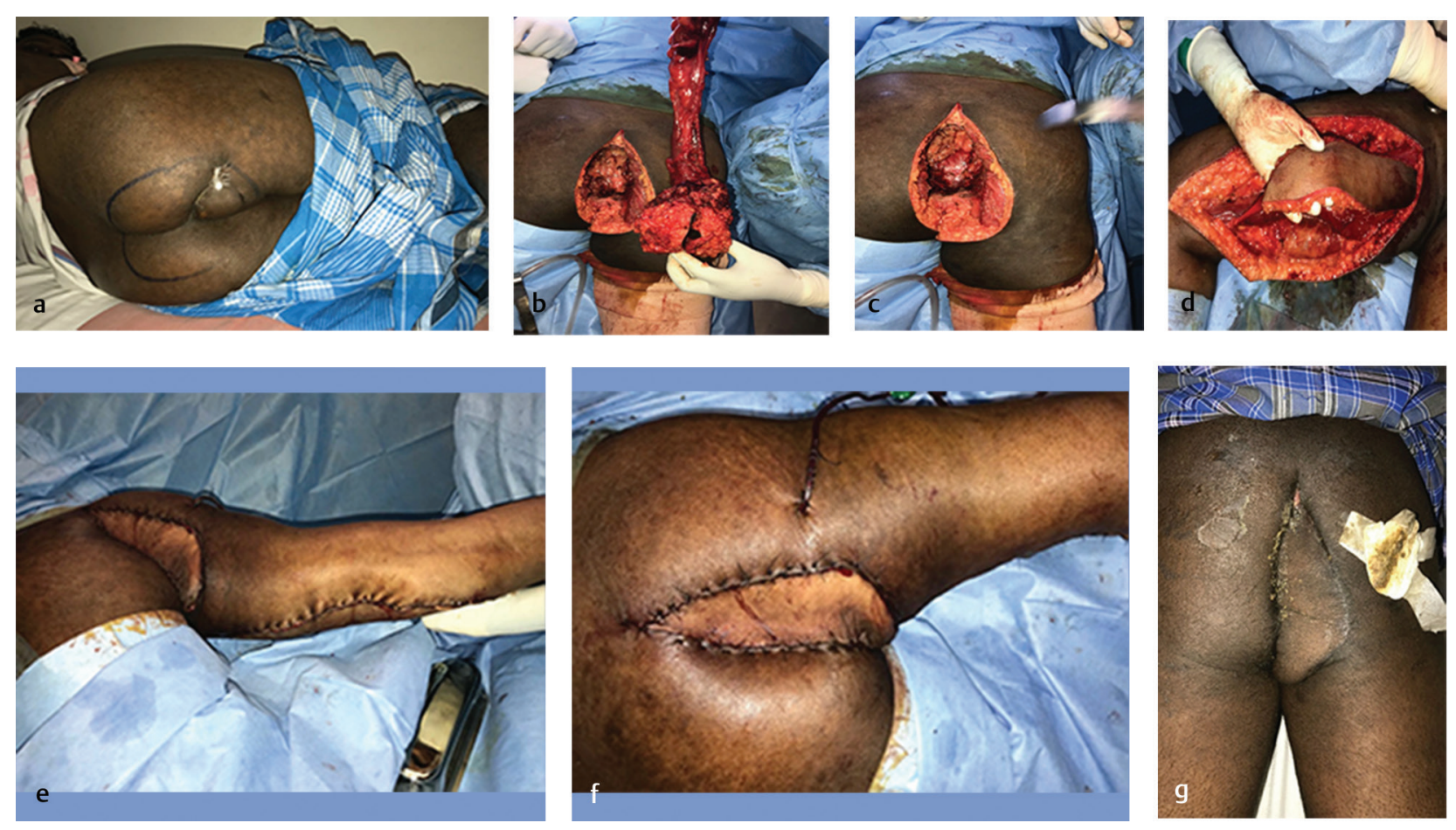

Fig. 2 (a-c) A case of rectal cancer invading the sacral fascia, pelvic and soft tissue in the perianal region status post radiation therapy. (d) Unilateral gracilis myocutaneous flap harvested in supine, pedicled, tunneled, and sutured into the defect post resection in prone position after placing a mesh to protect the abdominal cavity. (e,f) Immediate postoperative status. (g) 2 months postoperative status.

VRAM flap reconstruction based on deep inferior epigastric artery, is an established standard and remains the workhorse flap for the large complex perineal defects since decades. ${ }^{4}$ The robust well-vascularized skin paddle and muscle bulk provided by this flap along with the ease of surgical technique are distinct advantages that make this flap to be preferred over other flaps by most reconstructive surgeons. ${ }^{5}$ However, this flap has its own limitations like abdominal wall weakness and tendency to herniate and cosmetically poorly placed scar. In patients who are more likely to have dual ostomies, placement of ostomies becomes technically more challenging. Even though anterolateral thigh flap provides the adequate muscle bulk it comes at the cost of significant functional disability. ${ }^{6}$

The gracilis myocutaneous flap was first described by Bartholdson and Hulten. ${ }^{7}$ Gracilis is the superficial most adductor muscle of thigh supplied predominantly by medial femoral circumflex artery with additional minor perforators from the obturator artery. Although pedicled gracilis flap is principally used in perineal reconstruction, its use extends to defects involving groin, thigh, external genitalia, and gluteal region. The distinct advantages of this unique flap include lack of donor site morbidity and adequate skin paddle with a constant neurovascular pedicle. It provides the option of harvesting bilateral flaps for larger defects. The added advantages of gracilis flap when used for perineal reconstruction include donor site from an area away from irradiated site, noninterference with the ostomy site. ${ }^{8}$ It does not compromise on abdominal wall tension or lower limb function unlike other flaps.
The use of gracilis flap for small perineal defects is well established and is commonly performed. ${ }^{9}$ But in our study we used gracilis flap for two patients who had a large 3D defect. Gracilis becomes an option if the VRAM flap is technically not available due to previous abdominal surgeries. In our patient gracilis myocutaneous flap provided sufficient vascularized tissue to reconstruct the large complex defect following pelvic exenteration for rectal cancers. With gracilis myocutaneous flap, abdominal wall integrity and possibility of herniation risk due to harvest of VRAM is technically avoided.

In series from Chong et al, the gracilis flap has been used for recontruction of large perineal and pelvic defects including complex perineal reconstruction following abdomino perineal resection (APR) and pelvic exenterations. ${ }^{6}$

Sharma et al recommends gracilis myocutaneous flaps as a viable option to provide the bulk and skin resurfacing for large perineal defects. ${ }^{1}$

\section{Disadvantages}

Since bulk offered by a single flap is less, need for bilateral flap for obliterating dead space exists. Vascularity of skin paddle over distal third of muscle is less reliable. This necessitates postoperative nursing care in prone position to prevent pressure injury, shearing, and necrosis of flap. Unfortunately it does not provide a stable interface to prevent herniation. A strong fascial component is missing unlike tensor fascia-lata flap or the anterolateral thigh flap. 


\section{Conclusion}

With advancement in the treatment options for rectal cancer patients, the survival rates are increasing. This forces reconstructive surgeons not only to think about reconstruction but to choose an option that has minimal morbidity to the patient without interfering with quality of life. Gracilis myocutaneous flap has its own place with unique advantage adding to the armamentarium of reconstructive options for complex perineal defects, thereby avoiding the morbidity associated with VRAM flap. It stands as a reliable alternative in patients where VRAM cannot be used, obviating the need for microsurgery.

\section{Conflict of Interest}

None.

\section{References}

1 Sharma RK, Parashar A. The management of perineal wounds. Indian J Plast Surg 2012;45(2):352-363

2 Temple L, Lindsay RL, Temple WJ, et al. Rectus myocutaneous flaps for primary repair for composite resection defects of the pelvis. Perspect Colon Rectal Surg 1993;6:171-176
3 Loessin SJ, Meland NB, Devine RM, Wolff BG, Nelson H, Zincke $\mathrm{H}$. Management of sacral and perineal defects following abdominoperineal resection and radiation with transpelvic muscle flaps. Dis Colon Rectum 1995;38(9):940-945

4 Touny A, Othman H, Maamoon S, Ramzy S, Elmarakby H. Perineal reconstruction using pedicled vertical rectus abdominis myocutaneous flap(VRAM).JSurg Oncol 2014;110(6):752-757

5 Buchel EW, Finical S, Johnson C. Pelvic reconstruction using vertical rectus abdominis musculocutaneous flaps. Ann Plast Surg 2004;52(1):22-26

6 Chong TW, Balch GC, Kehoe SM. Margulis V, Saint-Cyr M. Reconstruction of large perineal and pelvic wounds using gracilis muscle flaps. Ann Surg Oncol 2015;22(11):3738-3744

7 Bartholdson L, Hultén L. Repair of persistent perineal sinuses by means of a pedicle flap of musculus gracilis. Case report. Scand J Plast Reconstr Surg 1975;9(1):74-76

8 Bell JG, Weiser EB, Metz P, Hoskins WJ. Gracilis muscle repair of perineal hernia following pelvic exenteration. Obstet Gynecol 1980;56(3):377-380

9 Baek SM, Greenstein A, McElhinney AJ, Aufses AH Jr. The gracilis myocutaneous flap for persistent perineal sinus after proctocolectomy. Surg Gynecol Obstet 1981;153(5):713-716 